\title{
Object Detection System using Arduino and Android Application for Visually Impaired People
}

\author{
Yesha A. Karmakar \\ B.Tech Student \\ Madhuben \& Bhanubhai \\ Patel Women's Institute of \\ Engineering, New Vallabh \\ Vidyanagar \\ Gujarat, India
}

\author{
Krina A. Suthar \\ B.Tech Student \\ Madhuben \& Bhanubhai \\ Patel Women's Institute of \\ Engineering, New Vallabh \\ Vidyanagar \\ Gujarat, India
}

\author{
Ayushi Y. Vadwala \\ B.Tech Student \\ Madhuben \& Bhanubhai \\ Patel Women's Institute of \\ Engineering, New Vallabh \\ Vidyanagar \\ Gujarat, India
}

\author{
Nirali Pandya \\ Assistant Proffesor \\ Madhuben \& Bhanubhai \\ Patel Women's Institute of \\ Engineering, New Vallabh \\ Vidyanagar \\ Gujarat, India
}

\begin{abstract}
This paper explores the working principle and applications of an Arduino board together with Android. This also explores on how they can be used to create an object detection system which can give response in speech format. At present many blind assistive systems have been implemented but here in this paper a proposed system which is android based and it detects the object in front of the blind person. The blind person is directed through English voice commands generated by the application according to the obstacle position. Our proposed system will be beneficial for applications such as assistive robots for people with visual disabilities or in industrial applications such as work robots.
\end{abstract}

\section{Keywords}

Arduino, Android, Object Detection, PIR sensor

\section{INTRODUCTION}

Vision is the most important part of human body processes as $83 \%$ of information is taken through sight by humans. World Health Organization (WHO) in 2011 estimates that there are 285 billion visually impaired people in the world, approximately $13 \%$ of which are blind and rest are with low vision. The old-fashioned mobility aids for people with visual disabilities are the walking cane (also called white cane or stick) and guide dogs. There are some drawbacks for these old-fashioned means such as necessary skills and training phase, range of motion and very little information conveyed. With the swift progresses of present technology, both in hardware and software front has conveyed latent to provide intelligent navigation capabilities. In recent times there has been a lot of Electronic Travel Aids (ETA) [1] designed and devised to help the blind navigate independently and safely.

Kumar, M. N., \& Usha, K. (2013) suggested the design and architecture of a new concept of Smart Electronic Travel Aid Stick for visually impaired people. The benefit of the scheme stays in the fact that it is prove to be a cheap solution to millions of blind people worldwide. [2]

Bahadir, S. K., Koncar, V., \& Kalaoglu, F. (2012) proposed an innovative wearable object detection system entirely embedded into textile structures, which enables detection of obstacles for visually impaired people, has been developed. The working of the proposed technology is based on two main tasks: sensing the surroundings and detection of obstacles with the help of sonar sensors. This system is able to identify obstacle's position within the detection range. It is easily worn as a garment that is flexible, lightweight and comfortable for human body as well as washable. The proposed smart clothing system could become united part of visually impaired people's lifestyle. [3]

Tanveer, M. S. R., Hashem, M. M. A., \& Hossain, M. K. (2015) implemented a system for helping the blind person in navigating himself. The final result of our approach is a blind navigation and tracking system with a flexible architecture. The blind assistive device that is Eyemate for Blind along with android application is useful for a blind person. In case of emergencies the voice call can be placed by the blind person. The system involves a BlindTracker application which is applicable for tracking the current location of the blind person. [4]

Sakhardande, J., Pattanayak, P., \& Bhowmick, M. (2012) suggested a system whose basic aim of the design was to develop and implement an Obstruction Detection System for Visually Impaired People with the help of ultrasound sensor. The smart cane is an inexpensive alternative way of identifying assistive devices to develop and build a smart cane for the visually impaired to detect obstacles [5]

The proposed system can be designed to take of form of a detachable and portable device, which can be unconditionally mounted on a simple white cane or blind stick. This requires a clear vision of the desired system goals. For the use of a blind person it can also be made in the form of necklace and hang it around the neck.

\section{SYSTEM ARCHITECTURE}

The figure below shows the proposed design of a system. System architecture is the theoretical and conceptual model that describes the structure, behaviour and more on the views of a system. It could comprise system components. In embodiment, it also provides the external evident properties of those components and the correlation between them. The Component of the system consist of speech to text conversation through android application i.e. software part and object detection using PIR sensor i.e. hardware part as shown in figure.

In Speech to Text conversion, an uttered word can be convert in to text form using predefined methods [12].This converted text can be used in various functions like making call, send message, set alarms, and many more which was suggested by the research and survey[13]. In our case, user must have to command "Connect Arduino" via speech, this command will compel Android application to connect with the Arduino. Then later whenever PIR sensor will detect something in front of it will generate notification alert that "Object detected". Android smart phone is connected to the Arduino using Bluetooth. The PIR sensor is connected to the Arduino board. When the user is new they must have to turn on Bluetooth and 
pair their smart phone with HC-05 IC. Then after the connection is successful, it will sense the object with the help of the PIR sensor and will alert the user via speech which says "object detected". An additional acknowledgement is shown with the help of LED. It lights on when object is detected and is approximately one feet away.

The proposed design for smart object detection manifestly consists of two units:

$$
\begin{array}{ll}
\text { i. } & \text { Software } \\
\text { ii. } & \text { Hardware }
\end{array}
$$

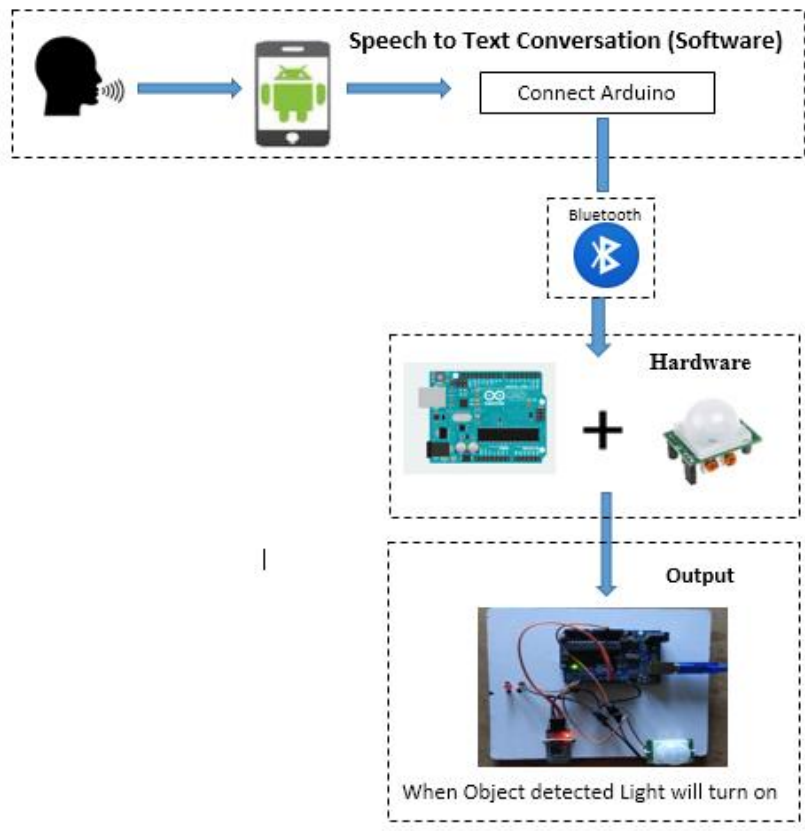

Fig 1. System Architecture

\section{HARDWARE}

Hardware consist of the following modules:

\subsection{Arduino}

Arduino is an open-source podium used for building electronics projects. Arduino comprises of both a physical programmable circuit board (often referred to as a microcontroller) and a portion of software, or IDE (Integrated Development Environment) that runs on the system, used to write and upload system code to the physical board.

The Arduino platform has turn into quite popular with people just starting out with electronics, and for good reason. Unlike most previous programmable circuit boards, the Arduino does not need a separate piece of hardware (called a programmer) in order to load new code onto the board - you can simply use a USB cable. Additionally, the Arduino IDE uses a simplified version of $\mathrm{C}++$, making it easier to learn to program. Finally, Arduino provides a standard form factor that breaks out the functions of the micro-controller into a more accessible package. The Uno is one of the more popular boards in the Arduino family and a great choice for beginners. [6]

A pre-assembled Arduino board includes a microcontroller, which is programmed using Arduino programming language and the Arduino development environment. In essence, this platform provides a way to build and program electronic components. Arduino programming language is a simplified from of $\mathrm{C} / \mathrm{C}++$ programming language based on what
Arduino calls "sketches," which use basic programming structures, variables and functions. These are then converted into a $\mathrm{C}++$ program. [7]

\subsection{Bluetooth}

Bluetooth is a kind of wireless communication. Bluetooth is use to transfer voice and data at high speed using radio waves. Bluetooth is intended for short range radio communication between many different types of devices like Mobile phone, computers and other electronics. It has a range of around 10 meters and transmission rate of 3 Mbps. [8]

\section{HC-05}

Figure 2 shows the HC-05 module which is a Bluetooth module. It is easy to use Bluetooth Serial Port Protocol (SPP) module. It is propose for transparent wireless serial connection setup. The Bluetooth module HC-05 is a master/slave module [9]. By default the factory setting is slave. The function of the module (Master or Slave) can be configured only by at commands. The slave modules cannot initiate a connection to another Bluetooth device, but can accept connections. Master module can initiate a connection to other devices. [10]

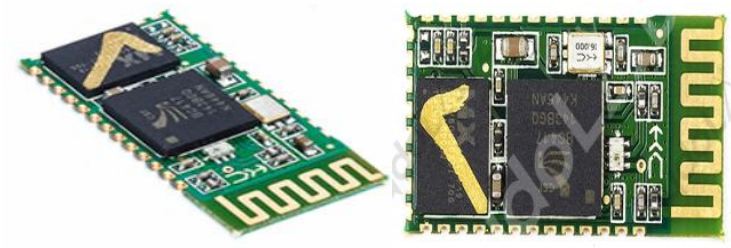

Fig 2. HC-05

HC-05 Specification:

- Bluetooth protocol: Bluetooth Specification v2.0+EDR

- Frequency: $2.4 \mathrm{GHz}$ ISM band

- Modulation: GFSK(Gaussian Frequency Shift Keying)

- Emission power: $\leq 4 \mathrm{dBm}$, Class 2

- Sensitivity: $\leq-84 \mathrm{dBm}$ at $0.1 \%$ BER

- Speed: Asynchronous: 2.1Mbps(Max) / 160 kbps, Synchronous: $1 \mathrm{Mbps} / 1 \mathrm{Mbps}$

- Security: Authentication and encryption

- Profiles: Bluetooth serial port

- Power supply: +3.3VDC 50mA

- Working temperature: $-20 \sim+75$ Centigrade

- Dimension: $26.9 \mathrm{~mm}$ x $13 \mathrm{~mm}$ x $2.2 \mathrm{~mm}[11]$

\subsection{PIR Sensor}

PIR sensor is the acronym of Passive Infrared Sensor. It measures the amount of infrared energy radiated by substances in front of it. They does not emit any kind of radiation but intellects the infrared waves radiated or reflected by objects.

The heart of a PIR sensor is a solid state sensor or an array of such sensors made from pyro-electric materials. Suitable lenses are fixed at the front of the sensor to focus the incoming radiation to the sensor face. Whenever an object or a human passes across the sensor the strength of the incoming radiation with respect to the background changes. As a consequence the energy generated by the sensor also changes. Suitable signal conditioning circuits convert the energy change to a suitable voltage output.

\section{Possible outputs from PIR sensor module}

The PIR sensor module has got only one digital output mode. So it has only 2 possible output values - either a HIGH or a 
LOW. By default standards, when there is no object inside the range of PIR sensor it outputs a LOW value or $0 \mathrm{~V}$ at output. When an object is identified inside the range of PIR sensor it immediately outputs a $\mathrm{HIGH}$ value or $+5 \mathrm{~V}$ at output.

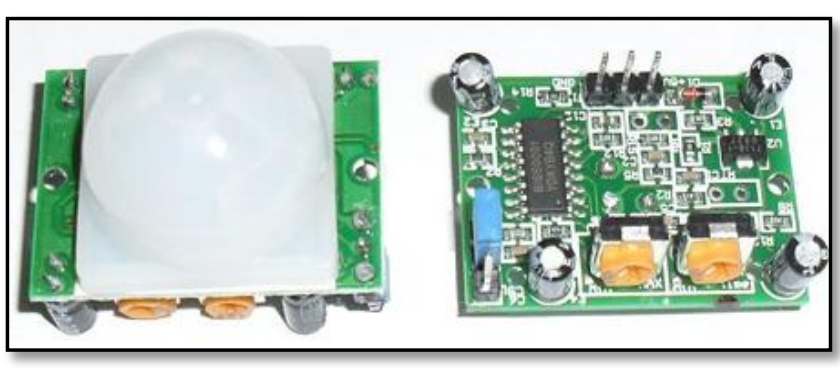

Fig 3. PIR sensor

\section{PERKS OF THE SYSTEM:}

1) This is very beneficial for visually impaired person.

2) In future, this can escalated in 360 degree by adding 6 PIR sensors or else using ultrasonic sensors to detect any object in front of the user.

3) This system can be used in industries for detecting people or objects in the restricted area.

\section{CONCLUSION}

This paper proposed the design and architecture of a new concept of object detection system for blind people using Arduino and Android. The plus point of the system lies in the datum that it can prove to be a very low cost solution to millions of blind person worldwide. The proposed combination of various working units makes a real-time system detect the object in front of the user and provide the alert feedback, thus making navigation more safe and secure.

\section{REFERENCES}

[1] Kumar, A., Patra, R., Manjunatha, M., Mukhopadhyay, J., \& Majumdar, A. K. (2011, January). An electronic travel aid for navigation of visually impaired persons. In Communication systems and networks (COMSNETS), 2011 third international conference on (pp. 1-5). IEEE.

[2] Kumar, M. N., \& Usha, K. (2013). Voice Based
Guidance and Location Indication System for the Blind Using GSM, GPS and Optical Device Indicator. International Journal of Engineering Trends and Technology (IJETT), 4(7), 3083-3085.

[3] Bahadir, S. K., Koncar, V., \& Kalaoglu, F. (2012). Wearable obstacle detection system fully integrated to textile structures for visually impaired people. Sensors and Actuators A: Physical, 179, 297-311.

[4] Tanveer, M. S. R., Hashem, M. M. A., \& Hossain, M. K. (2015, December). Android assistant EyeMate for blind and blind tracker. In Computer and Information Technology (ICCIT), 2015 18th International Conference on (pp. 266-271). IEEE.

[5] Sakhardande, J., Pattanayak, P., \& Bhowmick, M. (2012). Smart cane assisted mobility for the visually impaired. World Academy of Science, Engineering and Technology, 70.

[6] Arduino- https://learn.sparkfun.com/tutorials/what-is-anarduino

[7] Arduinohttps://www.techopedia.com/definition/27874/arduino

[8] Jayantilal, S. H. Interfacing of AT Command based HC05 Serial Bluetooth Module with Minicom in Linux.

[9] Arduino-https://arduino-info.wikispaces.com/BlueToothHC05- HC06-Modules-How-To.html

[10] Gaikwad, P. V., \& Kalshetty, Y. R. (2015). Bluetooth Based Smart Automation System Using Android. International Journal of Science and Research.

[11] Bluetooth-https://www.rhydolabz.com/wirelessbluetooth-ble-c-130_132/hc05-bluetooth-modulemasterslave-p-1169.html

[12] Ayushi Y Vadwala, Krina A Suthar, Yesha A Karmakar and Nirali Pandya. Survey paper on Different Speech Recognition Algorithm: Challenges and Techniques. International Journal of Computer Applications 175(1):31-36, October 2017.

[13] Ms.Ayushi Y. Vadwala, Ms.Krina A. Suthar, Ms.Yesha A. Karmakar, P. N. P. (2017). Intelligent Android Voice Assistant - A Future Requisite. International Journal of Engineering Development and Research, 5(Issue 3), 337 339 . 